\title{
Envejecer en casa con teleasistencia en España. Un análisis del discurso
}

\author{
Aging at home with telecare in Spain. A dicourse analysis
}

Juan C. Aceros ${ }^{1}$

Maria Tereza Leal Cavalcante ${ }^{2}$

Miquel Domènech ${ }^{3}$

${ }^{1}$ Vicerectoría de

\begin{abstract}
Caring for the elderly is turning to forms of community care and home care. Telecare is one of those emergent modalities of caring. This article will explore the meanings that older people give to the experience of staying at home in later life by using telecare. Discourse analysis is used to examine a set of focus groups and interviews with telecare users from different cities of Catalonia (Spain). The outcomes include three interpretative repertoires that we called: "Aging at home", "normal aging" and "unsafe aging". For each repertoire we examine how the permanence of older people in their homes is accounted, and which role telecare plays in such experience.
\end{abstract}

Key words Home care, Technology, Older people, Aging, Discourses
Resumen El cuidado de las personas mayores está dando un giro hacia modalidades de atención comunitaria y domiciliaria. La teleasistencia es una de dichas modalidades. Este artículo se propone explorar los significados que las personas mayores asocian a la experiencia de permanecer en el hogar a edades avanzadas con ayuda de la teleasistencia. Se utiliza el análisis del discurso para examinar un conjunto de grupos de discusión con $y$ de entrevistas a usuarios de teleasistencia de diferentes ciudades de Cataluña (España). Como resultado se presentan tres repertorios interpretativos denominados: "envejecimiento en casa", "envejecimiento normal" y "envejecimiento inseguro". Para cada uno se examina la forma como describen la permanencia de las personas mayores en sus hogares, así como el papel que la teleasistencia juega en dicha experiencia.

Palabras clave Asistencia domiciliaria, Tecnología, Personas mayores, Envejecimiento, Discursos 


\section{Introducción}

La atención socio-sanitaria de las personas mayores está cambiando en la dirección de modalidades de cuidado comunitario y domiciliario que promueven el "envejecimiento en el lugar" (aging in place $)^{1,2}$. Este modelo propende por la permanencia de las personas en su entorno de vida habitual a edades avanzadas, tanto como sea posible ${ }^{3}$. La idea es relativamente nueva, pero goza de una amplia aceptación ${ }^{4}$. En España, se están instaurando numerosos servicios que responden al ideal del envejecimiento en el lugar (centros de día y de noche, pisos tutelados, etc.). El aumento de estas formas de atención socio-sanitaria hace necesario un examen de su funcionamiento, de sus efectos y del significado que tienen para sus beneficiarios $^{4,5}$. Este artículo se interesa por el significado de la teleasistencia, un servicio que implementa soluciones técnicas para envejecer exitosamente en los lugares de residencia habitual.

La forma más extendida de teleasistencia en España incluye un conjunto de equipos instalados en el hogar (una terminal telefónica, un dispositivo "manos libres", detectores de caídas, etc.), que en caso de emergencia permiten enviar una alarma a una central desde donde se pueden movilizar recursos para auxiliar al usuario ${ }^{6}$. Los promotores y proveedores de teleasistencia sostienen que con la ayuda de estas tecnologías es posible asegurar el bienestar de los mayores, promover su independencia y reducir los ingresos a instituciones socio-sanitarias ${ }^{7}$. Por ejemplo la Cruz Roja de Cataluña, una de las instituciones pioneras en la provisión de teleasistencia, afirma que este servicio "promueve la autonomía de las personas mayores (...) garantizando la seguridad y tranquilidad de los usuarios y de sus familiares". La Ley 34 de 2006 (o "Ley de dependencia"), insiste en este mismo argumento cuando contempla la teleasistencia como uno de sus "servicios de promoción de la autonomía personal”.

Diferentes trabajos muestran que las personas mayores tienen una actitud muy favorable tanto hacia el envejecimiento en casa ${ }^{9-11}$ como hacia la teleasistencia ${ }^{5,12}$. Sin embargo, aún se sabe poco sobre el sentido que envejecer en casa con teleasistencia tiene para los mayores. El objetivo de este artículo es acercarse a esta cuestión. La pregunta que pretende responder es: ¿Cómo interpretan las personas mayores su permanencia en el hogar a edades avanzadas con ayuda de servicios de cuidado remoto? Responder a esta pregunta puede contribuir a la formulación de políticas públicas que contribuyan mejor a la salud del creciente colectivo de personas mayores.

\section{Métodos}

Este artículo presenta un acercamiento cualitativo a la comprensión que los mayores tienen de envejecer en casa con teleasistencia. Concretamente, emplea el análisis del discurso, un método interpretativo que Íñiguez y Antaki ${ }^{13}$ definen así: "Un discurso es un conjunto de prácticas lingüísticas que mantienen y promueven ciertas relaciones sociales. El análisis consiste en estudiar cómo estas prácticas actúan en el presente manteniendo y promoviendo estas relaciones". Entre las distintas tradiciones de $\mathrm{AD}$, se ha optado por el análisis de repertorios interpretativos ${ }^{14,15}$. Esta perspectiva entiende que cualquier objeto del mundo puede construirse discursivamente de diferentes maneras, para cumplir funciones variadas. Cada versión es producida movilizando un repertorio interpretativo específico. Por "repertorio interpretativo" se entiende un sistema de significación compuesto por términos, descripciones y figuras del lenguaje que están convencionalmente relacionados entre sí y que son usados de una manera estilística y específica para caracterizar y evaluar objetos, acciones y eventos ${ }^{14,16}$.

Los datos provienen de dos investigaciones sobre teleasistencia en España en las que se utilizaron diferentes métodos cualitativos. Para los efectos de este artículo solo se han analizado los datos recogidos mediante grupos de discusión con y entrevistas semiestructuradas a adultos mayores (descartando datos obtenidos con trabajadores de teleasistencia, profesionales sociosanitarios, familiares y cuidadores). En los grupos de discusión participaron 19 personas mayores usuarias de un proveedor de teleasistencia privado. Para su selección se empleó el muestreo propositivo. La muestra incluyó personas de más de 65 años, de diferentes ciudades de Cataluña, con distintos tiempos de permanencia en el servicio. Aunque se procuró que los grupos estuvieran equilibrados en cuanto al género, la mayor parte de las participantes fueron mujeres; lo que se corresponde con el perfil de usuario de teleasistencia en España. Los grupos de discusión se realizaron en las instalaciones del proveedor que colaboraba con el estudio y tuvieron una duración de aproximadamente una hora. Para su realización se contaba con un guión con preguntas sobre usos y características de los dispositivos, beneficios y riesgos de la teleasistencia, opiniones sobre el servicio y espacios de la vida cotidiana. Los encuentros fueron grabados con la autorización de los participantes y fueron transcritos en su totalidad.

Para complementar la información recogida durante los grupos de discusión, se llevaron 
a cabo entrevistas individuales a 9 usuarios residentes en diferentes ciudades catalanas. Estos últimos fueron seleccionados por conveniencia y sus entrevistas se llevaron a cabo en el contexto de visitas domiciliarias durante las cuales se llevaba a cabo observación participante. Las entrevistas se realizaron con atención al mismo guión empleado en los grupos de discusión, y fueron igualmente grabadas y transcritas.

Para identificar los repertorios interpretativos, se siguió el procedimiento recomendado por Potter y Whetherell ${ }^{15}$. Los investigadores hicieron una lectura independiente del material que condujo a una primera codificación temática. Así, fue posible focalizar el análisis posterior en fragmentos relevantes organizados por tópicos. Este artículo se centra en uno de los temas: la casa como lugar para envejecer. La selección de fragmentos a ser incluidos en dicho tema fue inclusiva. Se tuvieron en consideración todos los segmentos de los que se sospechara una correspondencia temática, aún a riesgo de incluir algunos sobre los que los analistas no estaban seguros. Posteriormente, se volvió sobre los datos con el objetivo de completar y refinar la codificación. A partir de una serie de relecturas se identificaron patrones de variabilidad discursiva y su consistencia interna. La identificación de dichos patrones llevó finalmente a la delimitación de un conjunto de repertorios interpretativos sobre el objeto de interés del estudio.

\section{Resultados y discussión}

El examen de los datos evidencia que las personas mayores movilizan diferentes formas de comprender el envejecimiento en casa con dispositivos tecnológicos. En este sentido, se han identificado distintos repertorios. En lo que sigue exponemos tres de especial relevancia e interés. Los hemos llamado: "envejecimiento en casa", "envejecimiento normal" y "envejecimiento inseguro". Así mismo, se examina el significado que la teleasistencia tiene en cada uno de estos repertorios.

\section{Envejecimiento en casa}

El "envejecimiento en casa” resuena con los discursos oficiales sobre aging in place. Consta de tres componentes principales. En primer lugar, presenta una preferencia por el hogar como lugar para permanecer en la vejez. En el siguiente fragmento se aprecia dicho componente. En él, una usuaria habla sobre una situación vivida con uno de sus hijos:
(1)

E: [Su hijo] Vio que usted está bien. No pasó nada.

P1: Sí y entonces, bueno. Quiso que me fuera con ellos y que me estuviera unos días allá con ellos $y$ después digo: Mira a mi dejadme ir a mi casa que es donde estoy mejor. Es que parece mentira, jeh? [...] Aunque tengan un piso que es fantástico [...] pero yo [prefiero estar] en mi casa.

En segundo lugar, este repertorio ofrece argumentos a favor de permanecer en el hogar a edades avanzadas. Este elemento argumentativo se aprecia en fragmentos como el siguiente:

(2)

E: ¿Prefieren el aparato [de teleasistencia] que estar en una residencia?

P2: Yo más que una residencia preferiría tener una mujer en casa. Estás en tu casa y haces más o menos lo de siempre.

El "envejecimiento en casa" presenta la opinión de que es importante que los mayores conserven su forma de vida, dando continuidad a sus rutinas cotidianas, en un entorno conocido. Dichas rutinas incluyen el desarrollo de las actividades "domésticas" (cocinar, limpiar, ver televisión, etc.) de acuerdo con las normas, ritmos y objetivos que la persona mayor se da a sí misma. También comprende la realización de actividades sociales en la comunidad inmediata, en compañía de vecinos, amigos y familiares, aunque tal opción es mencionada con menos frecuencia.

Finalmente, este repertorio incluye un componente descriptivo, que asocia al hogar un conjunto de atributos favorables. Lo hace mediante comparaciones entre la casa y otros lugares (residencias, instituciones de salud y la casa de los hijos). Estos últimos escenarios, en el mejor de los casos, se describen como "buenos" lugares; pero como no lo suficientemente buenos como para estar en ellos por mucho tiempo. En el peor de los casos, son descritos como ambientes perniciosos y “deprimentes", generadores de efectos negativos sobre las personas (esto es común cuando se contrasta el hogar con las residencias). Por oposición a esos lugares, el domicilio propio es dibujado como un lugar familiar, acogedor y potenciador de la independencia personal.

El "envejecimiento en casa" valora la familiaridad del hogar, la sensación de "estar en casa" que produce, así como la "autonomía" de la persona mayor. En efecto, constituye discursivamente a los mayores como sujetos capaces de "organizarse la vida" por sí mismos mientras vivan en un entorno conocido y confortable. El significado y el valor del hogar como espacio para el envejecimiento se construyen mediante contrastes con 
otros lugares; sin embargo, cuando los hablantes movilizan este repertorio terminan postulando el carácter incomparable del domicilio propio. En este sentido, formulaciones de casos extremos ${ }^{17}$ como "no hay lugar como el hogar" o "como la casa no hay nada" son indicativas de que este repertorio está siendo utilizado. Por ejemplo, en (3) un grupo de usuarias discute si estarían dispuestas a vivir en la casa de sus hijos:

(3)

P5: ¡Oh! Si te quieren genial. Si no te quieren... P2: No. Es que aunque quieran, no querría ir. P3: Sí, mujer sí.

P2: ¡No!

P5: Como la casa no hay nada.

P2: Ya lo sé. Pero en mi casa. En casa de las hijas no. No quiero ir a mortificar a nadie.

En los datos, el "envejecimiento en casa" aparece cuando los usuarios tienen que exponer sus preferencias entre un número determinado de alternativas. El repertorio permite escoger entre ellas, optando por la que socialmente se considera más aceptable. Adicionalmente, permite rechazar las representaciones estereotípicas de la vejez y capacita al hablante para 1) reafirmar su condición de persona capaz y responsable de sí misma y 2) descartar la posibilidad de que se le describa como un "problema" o una "carga" para los demás.

\section{Envejecimiento normal}

El "envejecimiento normal" comparte con el discurso anterior su preferencia por el hogar como sitio para vivir en la vejez. Sin embargo, se distingue por su concepción del envejecimiento y por su orientación hacia el futuro. Con respecto a esta última cuestión, el "envejecimiento normal" trasciende la fijación en el presente que caracteriza a los demás repertorios. Los otros discursos operan sobre una foto fija de las condiciones de vida actuales. El "envejecimiento normal" mira más allá y permite al hablante anticipar cambios por venir. Así, en (4) una usuaria imagina un momento de la vida en la que "no pueda salir de casa":

(4)

P2: Yo cuando me lo pusieron [el servicio] me dijeron que que si quería pagar más cada mes, que pagaba y entonces me llamarían no se si cada día o cada cuanto, para tener compañía. [...] Pues yo dije: ;Ay no no no! De momento yo no lo necesito [...] Y dije que si alguna vez me pasaba eso, que no pudiera salir de casa, yo que se... entonces podría pagar ese servicio, pero en principio no.
Este repertorio cuenta con una teoría lega sobre lo que algunos hablantes consideran el desarrollo humano "normal". La misma recuerda la mirada biomédica y su énfasis en el envejecimiento como proceso biológico degenerativo ${ }^{18}$. En fragmentos como el siguiente encontramos indicios del uso de dicha teoría:

(5)

E1: Y sus hijos, [...] ¿están contentos [con la teleasistencia]?

P2: Hombre, ;claro que están contentos! Porque saben que es una ayuda muy grande la de eso. [...] Porque me dicen: también puedes irte a una residencia. Pero ya me lo pensaré cuando no pueda más. Mi cabeza ... a lo mejor, claro que tenemos que ir a parar, porque todo el mundo va, pero de momento, mientras que pueda vivir ... yo no salgo de mi casa.

La idea principal del "envejecimiento normal" es que el proceso de envejecimiento viene acompañado de un conjunto de pérdidas cuya acumulación conduce a una "vejez dependiente"18 incompatible con la permanencia en casa. Tal razonamiento está implícito en frases como "en casa mientras pueda"; pero también puede exhibirse más visiblemente, sobre todo en descripciones que la persona hace de sí misma o de otras personas de edad avanzada. Esto es lo que ocurre en (5), cuando la usuaria menciona su "cabeza" (en lo que puede interpretarse como una referencia a un deterioro cognitivo por venir, o ya en proceso) o sostiene que "todo el mundo va" a experimentar los efectos del "envejecimiento normal".

Las descripciones del ciclo de vida "normal" construyen una imagen de la persona de edad avanzada como alguien que carece de las capacidades y/o los recursos para dirigir su propia vida y cuidar de sí mismo. Así pues, este repertorio resuena con representaciones sociales estereotípicas que asocian este momento de la vida con el declive, la enfermedad y la dependencia. La imagen deficitaria de la vejez que articula este repertorio también incluye, en ocasiones, referencias a relaciones sociales débiles que no pueden asegurar un adecuado cuidado de la persona mayor. El siguiente fragmento es un ejemplo. En él, el entrevistador pregunta a un grupo su opinión sobre el envejecimiento en casa:

(6)

E: ¿Estáis de acuerdo con esto?

P5: Eso no lo vemos muchas. Porque yo también no me gustaría irme [de mi casa]. Pero es lo que digo si no puedo pagar una mujer [una cuidadora], y los hijos están trabajando, tienen sus hipotecas y todo; pues, no pueden responder por mí. 
El hallazgo de este repertorio indica que entre los mayores existen definiciones opuestas y contradictorias del envejecimiento y la vejez. En un extremo, el "envejecimiento en casa" resuena con discursos oficiales desde los que se están definiendo formas novedosas de envejecimiento normativo ${ }^{19}$. Estas nuevas normatividades consideran que el "buen envejecimiento" es aquel que se acompaña de altas tasas de actividad y participación social, y que ocurre en lugares familiares. En el otro extremo, el "envejecimiento normal" asocia la vulnerabilidad, la enfermedad y la dependencia a la vejez. Cuando las personas utilizan el repertorio de la "normalidad" se aplican a sí mismas estas imágenes estereotípicas. Así, procuran presentarse públicamente como sujetos realistas que asumen y aceptan la identidad de persona-que-envejece. Las personas también emplean este repertorio para discutir con otros los límites del "envejecimiento en casa". En este sentido, los datos sugieren que la actitud positiva hacia el aging in place no necesariamente se mantiene a toda costa. Envejecer en casa puede concebirse como una decisión que se toma hasta nuevo aviso, a espera de lo que pase en el futuro.

\section{Envejecimiento inseguro}

Este repertorio es llamativo por su caracterización del hogar. La literatura psicológica suele resaltar el carácter restaurador de la casa, a la cual las personas asocian un conjunto de significados positivos ${ }^{20-22}$. Nuestro estudio constata ese mismo hecho, especialmente en el repertorio de "envejecimiento en casa"; sin embargo, también documenta una mirada menos auspiciosa. El “envejecimiento inseguro", en efecto, se estructura alrededor de la idea de la casa como entorno peligroso. Por ejemplo, en (7) las usuarias hablan de accidentes sufridos en el hogar:

(7)

P1: Eso le pasó a Laia, juy! [...] ¡Vinieron los bomberos y todo! [...] Con las escaleras esas tan altas, tuvieron que entrar porque ella...

P3: ¡Ah! ¡Que estaba en el balcón cuando cayó! ¡En el balcón!

P1: [...] Se rompió el femur en la misma casa, ¿h? Pues bueno. La llave la tenía en la cerradura, no podían entrar [...] y entraron hasta, por el balcón. Y ella en el suelo.

En fragmentos como este, la casa se cuestiona como lugar seguro y protector, y se interpreta el envejecimiento en el hogar desde la lógica de los accidentes y los riesgos ${ }^{23}$. Dicha lógica se moviliza especialmente cuando las personas refieren los motivos de contratación de la teleasistencia, y cuando hablan sobre su forma de emplear los dispositivos de alarma. En dichos contextos de enunciación, los hablantes realizan un análisis de riesgos en torno a tres temas. El primero remite a la naturaleza temporal del riesgo y resalta momentos del día en los que la persona mayor corre más peligro. Diversos hablantes coinciden en apuntar a la noche como el momento más riesgoso del día, como en (8):

(8)

P4: ... yo lo tengo puesto [el colgante] en la noche también [...] lo pongo en la mesa de noche. Pero muchos días me lo dejo porque si no me acuerdo al momento de levantarme... porque lo peligroso es cuando te levantas en la noche. Puedes caer. [...] Casi es más necesario de noche que de día [llevar el colgante], me parece a mi.

El segundo tema se refiere al carácter espacial del riesgo. Consiste en la identificación de lugares peligrosos para los mayores. Con frecuencia se hace mención de escaleras, jardines y patios; sin embargo, el lugar más mencionado es el lavabo y, en concreto, la bañera o la ducha:

(9)

E: ¿Cuándo se ducha también [usa el colgante]?

P1: Ya me han dicho que no me lo quite. Y lo levo, sí. Porque es el momento en el que más peligro tienes. Ya tengo una barra en la ducha, pues desde hace unos tres años tenía bañera y al final la cambié [...] puse ducha porque me costaba salir de la bañera.

Un tercer tema tiene que ver con actividades arriesgadas. Se trata de acciones mediante las que la persona mayor intenta mantener la casa en condiciones adecuadas de limpieza, orden y estética. En el siguiente ejemplo, el entrevistador pregunta a las participantes si desde que usan la teleasistencia hacen cosas que antes no hacían:

(10)

P2: La única cosa si, a ver: limpio un armario, me lo cuelgo [el colgante] [...] Normalmente no lo llevo puesto. Si limpio un sitio o tengo que colgar alguna cosa; ves... pues si. Enseguida me lo coloco, porque pienso: si caes de la silla o de la escalera; pues a ver...

Los distintos temas que componen el "envejecimiento inseguro" contradicen la mayor parte los estudios de los significados que el hogar tiene para las personas en general, y las personas mayores en particular (significados, frecuentemente positivos). Nuestros resultados se acercan más a los expuestos por Bofill Poch $^{24}$ quien ha documentado afectos negativos ligados al hogar 
en la vejez. En nuestro estudio, el "envejecimiento inseguro" se opone además al "envejecimiento en casa" y su imagen idealizada del hogar. La existencia de estas dos comprensiones contrapuestas de la casa como espacio para envejecer puede plantear a las personas mayores la paradoja de desear su permanencia en un lugar que resulta peligroso para ellas. Sin embargo, como se verá, el "envejecimiento inseguro" parece conectarse bastante bien con los otros dos discursos identificados.

El "envejecimiento inseguro" no es un repertorio completamente autónomo con respecto a los otros discursos. Este repertorio da por supuesto que las personas mayores desean envejecer en casa, a pesar de los riesgos que corren en ella. En este aspecto, comparte la valoración positiva que hemos encontrado en los demás repertorios. Por otro lado, comparte la visión carencial de la vejez que sostiene el "envejecimiento normal": postula que la vejez es un período vulnerable de la vida. Así, el "envejecimiento inseguro" parece funcionar como un discurso fronterizo que conecta a los otros repertorios. Una idea semejante ha sido planteada por López ${ }^{23}$ para quien la "seguridad es el problema que estructura el orden socio-técnico de la teleasistencia domiciliaria. La atención inmediata, la inclusión de la autonomía, el envejecimiento en casa y, finalmente, el cuidado tienen sentido cuando se basan en y son diseñados para la seguridad".

\section{La teleasistencia en los distintos repertorios}

Como puede apreciarse, cada repertorio plantea formas distintas de entender el envejecimiento en casa. Cada uno de ellos, además versiona de manera distinta la teleasistencia domiciliaria. La posibilidad de que existan un abanico de comprensiones el cuidado remoto ha sido estudiado por autores como Greenhalgh et al. ${ }^{25}$, quienes han hallado visiones que se debaten entre una aproximación favorable y optimista hacia la teleasistencia, y posicionamientos críticos que denuncian sus problemas y limitaciones. En nuestros datos, las posturas críticas no están representadas. En realidad los tres repertorios se muestran favorables a la teleasistencia.

$\mathrm{El}$ "envejecimiento en casa" incluye ideas que son coherentes con los discursos oficiales sobre las virtudes de la teleasistencia. Según dichos discursos, el servicio contribuye a mejorar la salud, prevenir la enfermedad, incrementar la calidad de vida y la satisfacción con el cuidado ${ }^{5}$. Tales ideas son integradas en el repertorio para dar fuerza retórica a la preferencia de los hablantes por el "envejecimiento en casa". Al contar con el servicio, el hablante puede justificar su elección y puede afirmar que cuenta con las condiciones requeridas para "envejecer en casa". De esta manera, le es más fácil persuadir a otros de que actúa racionalmente, considerando las opciones que aseguran su bienestar y disminuyen los riesgos que correría viviendo en casa sin ayudas técnicas. Así pues, la teleasistencia es, para el "envejecimiento en casa", un recurso retórico que sirve a los propósitos del hablante y que le ayuda a justificar su deseo de vivir autónomamente en un contexto familiar.

El "envejecimiento normal", dado su acento en lo temporal, plantea papeles provisionales para la teleasistencia. Se encuentran dos tendencias. Una de ellas es la de aquellos hablantes que se consideran personas "activas" (o que rechazan la categoría de "persona mayor"), pero que pronostican una situación carencial por venir. En estos casos, la utilidad de la teleasistencia queda en suspenso, hasta la llegada del futuro que la teoría lega del desarrollo "normal" predice. La persona, en este sentido, afirma contratar el servicio para cuando sea "mayor". La segunda tendencia es la de aquellas personas que se reconocen actualmente como "mayores" y que anticipan un aumento de su grado de dependencia. Para estas personas, la teleasistencia brinda cuidado y apoyo temporalmente, hasta el momento en el que consideren necesario desplazarse a una residencia.

Finalmente, el "envejecimiento inseguro" incluye descripciones de los mayores como sujetos frágiles, en constante riesgo de enfermar o de caer. En consecuencia, la teleasistencia aparece como solución a estos problemas, garantizando una ayuda rápida y oportuna, en situaciones de emergencia. Es así como la asistencia remota se asocia con una sensación de "seguridad", habitualmente encontrada en estudios sobre la percepción del servicio ${ }^{23}$. Adicionalmente, el "envejecimiento inseguro" realza los casos de aquellas personas que viven en hogares unipersonales y que sufren una situación que en ocasiones roza el abandono. De la conjunción entre la soledad y la vulnerabilidad resulta el riesgo de no contar con nadie en casa para obtener ayudar si se tiene un accidente o problema. Esto deriva en la comprensión de la teleasistencia como proveedor de "compañía", apoyo social y de una rápida atención en casos urgentes.

Así pues, en general, los repertorios antes descritos entienden la teleasistencia como proveedora de diferentes beneficios. Tales beneficios han 
sido identificados por otros estudios. Por ejemplo, López ${ }^{23}$ resalta la capacidad de la teleasistencia para ofrecer seguridad, compañía, cuidado y autonomía. Nuestros resultados se acercan a los de este autor, aunque sugieren que cada repertorio se focaliza en ventajas concretas: la teleasistencia para "sentirse en casa" y promover la “autonomía” son parte central del "envejecimiento en casa"; el acento puesto en la "seguridad" y la "compañía” son propios del "envejecimiento normal" y el "envejecimiento inseguro". Con atención a estos distintos valores, la teleasistencia se postula como una solución tecnológica a los problemas o necesidades tanto de las personas con altos niveles de "dependencia", como de aquellas que se sienten "autónomas".

\section{Conclusiones}

Los discursos sobre el envejecimiento en casa con teleasistencia son recursos culturales mediante los cuales las personas mayores pueden dar sentido a su experiencia cotidiana como usuarios del cuidado remoto. También pueden ser fuente de información relevante para la formulación de mejores políticas públicas orientadas al cuidado de las personas de edad avanzada. En efecto, los repertorios establecen lo que los potenciales beneficiarios de tales políticas consideran como formas de envejecimiento posible y deseable, brindando pistas sobre las necesidades que la política pública puede llegar a satisfacer.

Ahora bien, es necesario tener en cuenta que aunque cada uno de los repertorios es internamente consistente, en su conjunto están lejos de ofrecer indicaciones unívocas acerca del tipo de envejecimiento que puede considerarse como adecuado o exitoso. Por el contrario, vuelven incierto lo que es concebible como "buena" forma de envejecer: ¿Se trata de conservar el estilo de vida personal a toda costa o de explorar nuevas alternativas de bienestar? ¿Es deseable prolongar la estadía en casa todo lo posible, o sólo mientras las condiciones de salud lo permitan? ¿Se debe apostar por envejecer en un lugar que plantea un conjunto de peligros cotidianos?

La variabilidad discursiva que hemos encontrado - y especialmente las referencias al carácter condicionado del envejecimiento en casa, y a los riesgos asociados a dicha alternativa - contrasta con el acento que las políticas públicas actuales ponen en los aspectos positivos del aging in place. Articular la información que ofrece nuestro análisis a la formulación de planes y programas para el colectivo de mayores requeriría del diseño y puesta a punto de estrategias para abordar y gestionar los aspectos menos favorables de la permanencia en el domicilio a edades avanzadas. Esto es especialmente relevante para el caso de aquellos países en los que los sistemas de protección social son deficitarios y en los que el paradigma del aging in place puede servir de excusa para disminuir los recursos públicos destinados al cuidado de las personas mayores.

Las políticas basadas en el aging in place se acompañan de la esperanza (o la promesa) de que el envejecimiento poblacional no sobrecargará los sistemas de salud si cada persona mayor se vuelve capaz de cuidar de sí misma. Si esta apuesta por el auto-cuidado no está acompañada por el ofrecimiento de nuevos servicios y suficientes apoyos a los mayores puede llevar a situaciones de soledad y abandono, a la sobrecarga de los cuidadores, y al deterioro de la calidad de vida de las familias (sobre las que suele recaer la responsabilidad del cuidado de las personas de edad avanzada en España y los países latinoamericanos). La teleasistencia suele presentarse como una solución tecnológica a este tipo de inconvenientes. Sin embargo, sus beneficios quedan necesariamente restringidos a aquellas personas que pueden pagar por el servicio, o que reciben ayudas públicas para tal fin. Los resultados del presente trabajo se encuentran limitados necesariamente al colectivo de personas mayores que, por una razón $\mathrm{u}$ otra, pueden contar con tecnologías de cuidado remoto instaladas en sus hogares.

A la luz de los discursos analizados aquí, la teleasistencia parece contribuir a un mejor abordaje de los retos que plantea el envejecimiento poblacional a las sociedades contemporáneas. Estos servicios son entendidos por sus usuarios como herramientas para promover la "autonomía", disminuir las situaciones de "dependencia”, favorecer la permanencia en un entorno "familiar" y mantener a las personas mayores protegidas de los posibles "riesgos" de vivir solos en casa a edades avanzadas. Esto parece apoyar (o apoyarse en) los discursos oficiales mediante los cuales se presenta la teleasistencia como una confiable solución tecnológica a los problemas atribuidos al progresivo crecimiento de la población mayor de 65 años. Sin embargo, diversos autores han mostrado que las tecnologías de cuidado remoto no son necesariamente buenos aliados para la consecución de objetivos políticos ${ }^{26,27}$ (generando, en ocasiones, resultados inesperados o no deseados).

Para profundizar en los significados que la teleasistencia domiciliaria puede llegar a tener, así 
como para comprender mejor sus impactos en la salud colectiva, se requiere ir más allá del análisis de repertorios interpretativos. Futuros estudios han de atender las prácticas efectivas mediante las cuales la teleasistencia es introducida en la vida cotidiana de las personas mayores, examinando sus efectos en la calidad de vida de quienes optan por envejecer en casa. Los repertorios interpretativos son prácticas lingüísticas que están íntimamente conectadas con otras prácticas sociales ${ }^{13}$. Resulta prioritario explorar cómo tales prácticas se articulan con acciones concretas en contextos específicos de aplicación. Esto abre un espacio muy rico de investigación en el que es fundamental el reconocimiento de las realidades locales en las que la teleasistencia es utilizada. Existe un creciente número de investigaciones en este sentido en países europeos ${ }^{2,23,26,27}$. La situación es distinta en el entorno latinoamericano, donde los servicios de cuidado remoto son de más reciente implantación. Los discursos con los que los usuarios dan sentido a estos servicios, los beneficios y problemas que perciben en ellos, así como los retos a los que se enfrenta la implementación de la teleasistencia en Latinoamérica son cuestiones que futuras investigaciones podrán ayudar a esclarecer.

\section{Colaboradores}

El diseño de la investigación fue liderada por $\mathrm{M}$ Domènech. Los tres autores participaron en la recolección y análisis de los datos. La redacción del artículo fue liderada por JC Aceros. MTL Cavalcante y $\mathrm{M}$ Domènech colaboraron en la revisión y la edición final. 


\section{Referencias}

1. Rowles GD. Evolving images of place in aging and "aging in place". Generations 1993; 17(2):65-70.

2. López D, Sánchez-Criado T. Dwelling the Telecare Home. Place, Location and Habitability. Sp Cult 2009; 12(3):343-358.

3. Pastalan LA. Preface. In: Pastalan LA, editor. Aging in place: The role of housing and social supports. New York: Haworth Press; 1990. p. ix - xii.

4. Cutchin MP. The process of mediated aging-in-place:a theoretically and empirically based model. Soc Sci Med 2003; 57(6):1077-1090.

5. Agrell H, Dahlberg S, Jerant AF. Patients' perceptions regarding home telecare. Telemed J e-Health 2000; 6(4):409-415.

6. Cavalcante MTL, Aceros JC.; Domènech M. Teleasistencia pública en España: Consideraciones de sus efectos en los servicios sociales y sanitarios. Ed Soc. Rev Interv Soc 2012; 51:91-102.

7. IMSERSO. Normas Generales del Servicio de Teleasistencia Domiciliaria. Programa de Teleasistencia IMSERSO-FEMP. España: Ministerio de Sanidad y Política Social; 1999.

8. Creu Roja. Teleasistencia. Barcelona: Creu Roja; 2011.

9. Costa-i-Font J, Elvira D, Mascarilla O. "Ageing in place"? Exploring elderly people's housing preferences in Spain. Urban Stud 2009; 46(2):295-316.

10. Instituto de Mayores y Servicios Sociales. Encuesta de Mayores 2010 [Internet]. Madrid; 2010. [cited 2015 Jul 15]. Available from: http://www.imserso.es/imserso_01/documentacion/estadisticas/index.htm

11. Bofill Poch S. Cuidado formal e informal en Cataluña. Un análisis de la percepción de las mujeres mayores en relación a sus derechos y obligaciones en materia de cuidados y atención. Etnográfica 2006; X(2):263-281.

12. Hanson J, Percival J, Aldred H, Brownsell S, Hawley M. Attitudes to telecare among older people, professional care workers and informal carers: a preventative strategy or crisis management? Univers Access Inf Soc 2007; 6(2):193-205.

13. Iñiguez L, Antaki C. Análisis del discurso. Anthropos 1998; 177:59-66.

14. Whetherell M, Potter J. El análisis del discurso y la identificación de los repertorios interpretativos. In: Gordo A, Linaza J, editors. Psicologías, discursos y poder. Madrid: Visor; 1996.
15. Potter J, Wetherell M. Discourse Analysis. In: Smith JA, Harré R, van Langenhove L, editors. Rethinking methods in psychology. London: Sage; 1996. p. 80-92.

16. Potter J, Wetherell M. Discourse and social psychology. London: Sage; 1987.

17. Pomerantz A. Extreme case formulations: A way of legitimizing claims. Hum Stud 1986; 9(2-3):219-29.

18. Cerri C. Dependencia y autonomía: Una aproximación antropológica desde el cuidado de los mayores. Athenea Digit 2015; 15(2):111-140.

19. Walker A. Quality of life in old age in Europe. Growing older in Europe. New York: Open University Press; 2005.

20. Rubinstein R. Personal Identity and environmental meaning in later life. J Aging Stud 1990; 4(2):131-147.

21. Rowles G. A place to call home. In: Carstensen LL, Edelstein BA, editors. Handbook of Clinical Gerontology. New York: Pergamon; 1987. p. 335-353.

22. Shenk D, Kuwahara K, Zablotsky D. Older wome's attachments to their home and possessions. J Aging Stud 2004; 18(2):157-169.

23. López D. Securizing Care: Networks, immediacy and independence in a home telecare service. Athenea Digit 2009; 164(185):185-193.

24. Bofill Poch S. La injustícia en la vellesa. Rev d'etnologia Catalunya 2010; (35):70-87.

25. Greenhalgh T, Procter R, Wherton J, Sugarhood P, Shaw $\mathrm{S}$. The organising vision for telehealth and telecare: discourse analysis. BMJ Open 2012; 2(4):1-12.

26. Pols AJ. Care at a Distance. On the Closeness of Technology. Amsterdam: Amsterdam University Press; 2012.

27. Aceros JC, Pols J, Domènech M. Where is Grandma? Home telecare, good aging and the domestication of later life. Techn For Soc Chan 2015; 92:102-111.

Artigo apresentado em 21/07/2015

Aprovado em 27/01/2016

Versão final apresentada em 29/01/2016 
